\title{
BIFURCATION PHENOMENA FOR AN OXIDATION REACTION IN A CONTINUOUSLY STIRRED TANK REACTOR. II DIABATIC OPERATION.
}

\author{
M. I. NELSON ${ }^{1}$ and H. S. SIDHU ${ }^{2}$
}

(Received 21 October, 2002; revised 7 June, 2003)

\begin{abstract}
We extend an investigation into the bifurcation phenomena exhibited by an oxidation reaction in an adiabatic reactor to the case of a diabatic reactor. The primary bifurcation parameter is the fuel fraction; the inflow pressure and inflow temperature are the secondary bifurcation parameters. The inclusion of heat loss in the model does not change the static steady-state bifurcation diagram; the organising centre is a pitchfork singularity for both the adiabatic and diabatic reactors. However, unlike the adiabatic reactor, Hopf bifurcations may occur in the diabatic reactor. We construct the degenerate Hopf bifurcation curve by determining the double-Hopf locus. When the steady-state and degenerate Hopf bifurcation diagrams are combined it is found that there are 23 generic steady-state diagrams over the parameter region of interest. The implications of these structures from the perspective of flammability in the CSTR are discussed.
\end{abstract}

\section{Introduction}

In an earlier paper [7] we investigated the steady-state multiplicity exhibited by the reaction of a fuel/air mixture in a continuously stirred adiabatic reactor. The chemical mechanism used was a modification of a scheme originally proposed to explain the occurrence of cool flames observed in the oxidation of hydrocarbons $[8,9]$. Interest in this mechanism was revived in the late 1980s and it has subsequently been studied in various contexts. An overview of this work is provided in [7].

Our modified scheme consists of an exothermic oxidation reaction proceeding by two consecutive reactions. In the first step a precursor species $\mathscr{F}$ generates a reactive intermediate species $\mathscr{B}$ in a first-order reaction. In the second step the fuel species $\mathscr{B}$

\footnotetext{
'School of Mathematics and Applied Statistics, The University of Wollongong, Wollongong, NSW 2522, Australia; e-mail: mnelson@uow.edu.au.

${ }^{2}$ School of Physical, Environmental and Mathematical Sciences, University College, University of New South Wales, Australian Defence Force Academy, Canberra 2600, Australia.

(C) Australian Mathematical Society 2004, Serial-fee code 1446-1811/04
} 
is oxidised through an exothermic reaction to an inert product $\mathscr{C}$. The oxidation step is a second-order reaction, being first order with respect to both reactants. It is assumed that all the heat output is associated with the second reaction. This scheme is represented as

$$
\begin{aligned}
\mathscr{F} \stackrel{k_{1}}{\rightarrow} \mathscr{B} & \left(Q_{1}=0\right), \\
\mathscr{B}+\mathscr{O}_{2} \stackrel{k_{2}(T)}{\longrightarrow} \mathscr{C} & \left(Q_{2} \neq 0\right) .
\end{aligned}
$$

We consider the scenario in which the activation energy of the first reaction is zero, that is, only the second step is responsive to temperature. The oxidation reaction then has the overall representation

$$
\mathscr{F}+\mathscr{O}_{2} \rightarrow \mathscr{C}
$$

The model consists of four ordinary differential equations (ODEs). The state variables are the concentrations of the three chemical species $\left(\mathscr{F}, \mathscr{B}, \mathscr{O}_{2}\right)$ and the reactor temperature $(T)$. As the rate constant for reaction (1.1) is temperature independent the equation for the concentration of the precursor species can be solved analytically. This reduces the order of the system from four to three. The stoichiometry of the oxidation step, reaction (1.2), can then be used to link the concentrations of oxygen $\left(\mathscr{O}_{2}\right)$ and the fuel species $(\mathscr{B})$ at time $t$. Thus one of these species can be eliminated from the model, reducing its dimension to two. In the adiabatic model a further reduction is possible as the remaining species concentration and the temperature at time $t$ are linked. The physical reason for this is that when there is no heat-loss at the reactor walls there is only one mechanism for removing heat from the system-outflow of the reaction mixture. This mechanism reduces the heat content and concentration linearly. Thus the adiabatic model is one dimensional and consequently oscillatory behaviour cannot occur.

In our earlier paper the one-dimensional adiabatic model was analysed using singularity theory [7]. Here we extend our investigation to the diabatic reactor. This model has an extra degree of freedom and sustained oscillations are now possible. We consider the bifurcation problem when the fuel fraction is the primary bifurcation parameter as this is the most important case from the perspective of fire-retardancy.

\subsection{Singularity theory for systems of two coupled ordinary differential equations} Gray and Roberts [4] have provided a user-friendly account of the bifurcations that can occur in a system of two ODEs containing at most three independent parameters. These systems are of the form

$$
\frac{\mathrm{d} x}{\mathrm{~d} t}=f(x, y, \lambda, \alpha, \beta),
$$




$$
\frac{\mathrm{d} y}{\mathrm{~d} t}=g(x, y, \lambda, \alpha, \beta),
$$

where $x$ and $y$ are the state variables, $\lambda$ is the primary bifurcation parameter and $\alpha$ and $\beta$ are secondary bifurcation parameters.

As explained in [7] the steady-state bifurcation diagram is obtained by determining the locus of the cusp, isola and double-limit point curves in physical parameter space. These are co-dimension one singularities. A point that is co-dimension one in singularity theory is co-dimension two from the perspective of bifurcation theory. This method divides parameter space into regions, each corresponding to a different steady-state diagram. The conditions for the cusp and isola singularities are [4]:

Cusp $f=g=\frac{\mathrm{d} \lambda}{\mathrm{d} x}=\frac{\mathrm{d}^{2} \lambda}{\mathrm{d} x^{2}}=0, \quad \frac{\mathrm{d}^{3} \lambda}{\mathrm{d} x^{3}} \neq 0, \quad \frac{\mathrm{d} \alpha}{\mathrm{d} \lambda} \neq 0$,

Isola $f=g=\frac{\mathrm{d} \lambda}{\mathrm{d} x}=\frac{\mathrm{d} \alpha}{\mathrm{d} \lambda}=0, \quad \frac{\mathrm{d}^{2} \alpha}{\mathrm{d}^{2} \lambda} \neq 0, \quad \frac{\mathrm{d}^{2} \lambda}{\mathrm{d} x^{2}} \neq 0$.

(The double-limit point singularity does not occur in our model.)

In two dimensions there are three co-dimension one dynamic singularities. These are: the double-zero eigenvalue locus, also known as the Bogdanov-Takens bifurcation [6]; the double-Hopf locus, where two Hopf points come together; and the generalised (or degenerate) Hopf bifurcation, also known as a Bautin bifurcation [6], where a "soft" Hopf bifurcation changes into a "hard" one (or vice versa); that is, when the first Lyapunov coefficient vanishes. The conditions for these are [4]:

Double-zero eigenvalue

$$
\begin{gathered}
f=g=\operatorname{det} \mathrm{Jac}=\operatorname{tr} \mathrm{Jac}=0, \\
\frac{\mathrm{d} \mathrm{tr} \mathrm{Jac}}{\mathrm{d} \lambda} \neq 0, \quad \mu_{2} \neq 0, \quad \frac{\mathrm{d}^{2} \lambda}{\mathrm{d} x^{2}} \neq 0, \quad \frac{\mathrm{d}^{2} \lambda}{\mathrm{d} x^{2}} \neq \infty
\end{gathered}
$$

Double-Hopf locus

$$
f=g=\operatorname{tr} \mathrm{Jac}=\frac{\mathrm{d} \operatorname{tr} \mathrm{Jac}}{\mathrm{d} \lambda}=0, \quad \frac{\mathrm{d}^{2} \lambda}{\mathrm{d} x^{2}} \neq 0, \quad \mu_{2} \neq 0 ;
$$

Generalised Hopf bifurcation

$$
f=g=\operatorname{tr} \mathrm{Jac}=\mu_{1}=0, \quad \frac{\mathrm{d} \operatorname{tr~Jac}}{\mathrm{d} \lambda} \neq 0, \quad \mu_{2} \neq 0,
$$

where $\mathrm{tr} \mathrm{Jac}$ and det Jac represent the trace and determinant of the Jacobian matrix (Jac) and $\mu_{i}$ is the $i$ th Lyapunov coefficient of a Hopf point.

In this paper we shall not be concerned with the distinction between 'soft' and 'hard' Hopf bifurcations. Furthermore, there are no double-zero eigenvalue bifurcations in the parameter region considered. Thus the co-dimension 1 Hopf bifurcation diagram is determined solely by the double-Hopf point locus. 
1.2. Limit cycles in Sal'nikov schemes Sal'nikov schemes typically generate very stiff limit cycles with very large amplitudes. In the vicinity of a Hopf point the behaviour of the limit cycles is often highly singular with the amplitude rising very rapidly over a very narrow region of the primary bifurcation parameter. Visually it often appears that there is a vertical branch of solutions $[2,3,10]$. The path-following program Auto is unable to track these periodic orbits as its numerical solution technique fails in these parameter regions. The maximum temperature on these limit cycles is often so large that it is physically meaningless. These excessive temperatures are due to the simplicity of the model. In reality at these high temperatures various endothermic processes occur, lowering the temperature. Such endothermic processes are not normally included in simplified chemical schemes.

\section{Model equations}

The chemistry involved in our model is outlined in Section 1. For a description of the model physics and the relationship between the model and experimental procedures we refer to our previous paper [7] which also includes the dimensional counterparts of (2.1)-(2.6). The dimensional variables are scaled using a reference temperature $\left(T^{*}=\right.$ $T / 298$ ), a reference concentration (the concentration of an ideal gas at atmospheric pressure and a temperature of $298 \mathrm{~K}$ ) and a timescale based upon Newtonian cooling. Full details are provided in our earlier paper [7]. There is a one-to-one relationship between our dimensionless variables and their dimensional counterparts. Hence we often write, for example, 'inflow pressure' rather than 'dimensionless inflow pressure'.

The system that we study is:

dimensionless precursor concentration

$$
\frac{\mathrm{d} \mathscr{F}^{*}}{\mathrm{~d} t^{*}}=q_{1}^{*} \alpha-\left(q_{1}^{*}+q_{2}^{*}\right) \mathscr{F}^{*}-A_{1}^{*} \mathscr{F}^{*},
$$

dimensionless fuel concentration

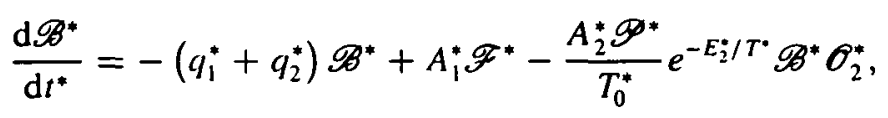

dimensionless oxygen concentration

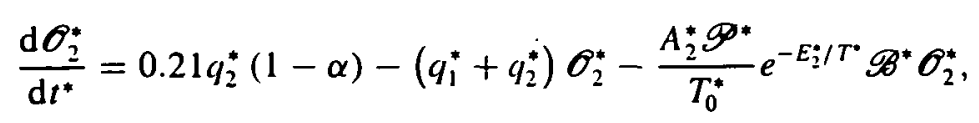

dimensionless temperature

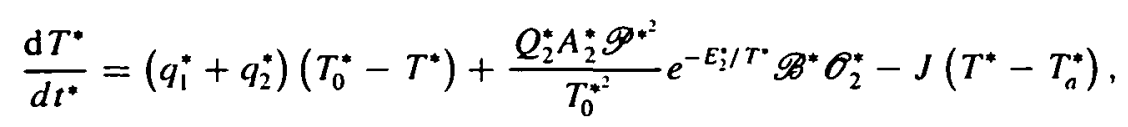


dimensionless initial conditions

$$
\begin{gathered}
\mathscr{F}^{*}(0)=\mathscr{B}^{*}(0)=\mathscr{O}_{2}^{*}(0)=0, \\
T^{*}(0)=\frac{\left(q_{1}^{*}+q_{2}^{*}\right) T_{0}^{*}+J T_{a}^{*}}{q_{1}^{*}+q_{2}^{*}+J} .
\end{gathered}
$$

The adiabatic case considered previously corresponds to the choice $J=0$. In (2.3) the number 0.21 is the fraction of oxygen in the air flowing through the reactor [7].

We assume that prior to the commencement of the experiment the entire system is first flushed with an inert species at temperature $T_{0}^{*}$. Thus the initial concentration of the chemical species in (2.5) is zero whilst the initial temperature of the reactor in (2.6) is the corresponding steady-state solution.

The experimentally controllable parameters are the reactant composition $(\alpha)$, the inflow pressure $\left(\mathscr{P}^{*}\right)$, the inflow temperature $\left(T_{0}^{*}\right)$, the inflow rates $\left(q_{1}^{*}\right.$ and $\left.q_{2}^{*}\right)$, and the vessel wall temperature $\left(T_{a}^{*}\right)$. The reactant composition parameter $(\alpha)$ is the fraction of the total concentration comprised by the pre-cursor species $\mathscr{F}$. The noncontrollable parameters are the: rate constant for the decay of the precursor $\left(A_{1}^{*}\right)$; the pre-exponential factor, activation energy and exothermicity for the oxidation reaction ( $A_{2}^{*}, E_{2}^{*}$ and $Q_{2}^{*}$ respectively); and a dimensionless constant controlling the amount of heat-transfer in the system $(J)$.

In what follows we assume that the inflow temperature $\left(T_{0}^{*}\right)$ is equal to the temperature of the walls of the reactor $\left(T_{a}^{*}\right)$. We also assume the inflow rates are equal $\left(q_{1}^{*}=q_{2}^{*}\right)$. We take the fuel fraction as the primary bifurcation parameter. The inflow pressure and the inflow temperature are the secondary bifurcation parameters.

2.1. Reduction of the model From (2.1) it follows that the precursor species $\left(\mathscr{F}^{*}\right)$ obtains the following steady-state value exponentially in time:

$$
\mathscr{F}_{s: s}^{*}=\frac{q_{1}^{*} \alpha}{q_{1}^{*}+q_{2}^{*}+A_{1}^{*}} .
$$

In [7] we showed that after an exponential decay of initial transience the concentration of the fuel species $\left(\mathscr{B}^{*}\right)$ is given by

$$
\mathscr{B}^{*}\left(t^{*}\right)=\frac{q_{1}^{*} \alpha A_{1}^{*}-0.21 q_{2}^{*}(1-\alpha)\left[q_{1}^{*}+q_{2}^{*}+A_{1}^{*}\right]}{\left(q_{1}^{*}+q_{2}^{*}\right)\left(q_{1}^{*}+q_{2}^{*}+A_{1}^{*}\right)}+\mathscr{O}_{2}^{*}\left(t^{*}\right)
$$

Under diabatic operation the steady-state behaviour of the four-dimensional system given by (2.1)-(2.4) reduces to the study of the following system:

dimensionless oxygen concentration

$$
\frac{\mathrm{d} \mathscr{O}_{2}^{*}}{\mathrm{~d} t^{*}}=0.21 q_{2}^{*}(1-\alpha)-\left(q_{1}^{*}+q_{2}^{*}\right) \mathscr{O}_{2}^{*}-\frac{A_{2}^{*} \mathscr{P}^{*}}{T_{0}^{*}} e^{-E_{2}^{*} / T^{*}} \mathscr{B}^{*}\left(t^{*}\right) \mathscr{O}_{2}^{*}
$$


dimensionless temperature

$$
\frac{\mathrm{d} T^{*}}{\mathrm{~d} t^{*}}=\left(q_{1}^{*}+q_{2}^{*}\right)\left(T_{0}^{*}-T^{*}\right)+\frac{Q_{2}^{*} A_{2}^{*} \mathscr{P} *^{2}}{T_{0}^{*}} e^{-E_{2}^{*} / T^{*}} \mathscr{B}^{*}\left(t^{*}\right) \mathscr{O}_{2}^{*}-J\left(T^{*}-T_{a}^{*}\right)
$$

with the algebraic relationship for $\mathscr{B}^{*}\left(t^{*}\right)$ given by (2.7).

2.2. Numerics The path-following software program Auto 97 [1] was used to obtain steady-state diagrams. In these the standard representation is used: solid lines are stable steady states; dotted lines are unstable steady states; squares are Hopf bifurcation points; open circles are unstable periodic orbits; and filled-in circles are stable periodic orbits. We investigate the flammability (ignitability) of our system. This corresponds experimentally to the establishment of a flame in the CSTR. Accordingly in our steadystate diagrams we plot the variation of the dimensionless temperature $\left(T^{*}\right)$ with the fuel fraction $(\alpha)$. When considering periodic solutions we plot the maximum value of the temperature on the limit cycle $\left(T_{m}^{*}\right)$ and for clarity we restrict the solution norm to only show limit cycles having a 'small' maximum temperature.

We frequently refer to Hopf bifurcation points on a steady-state diagram as (H1), (H2), etcetera. Here ( $\mathrm{H} 1)$ is the first Hopf point to be reached if one traces the steadystate curve, starting at the unique steady-state solution when there is no fuel in the system $(\alpha=0)$. We refer to Hopf points as being either fuel-lean, if $\alpha<0.174$, or fuel-rich, if $\alpha>0.174$. When $\alpha=0.174$ the concentrations of the precursor species and oxygen flowing into the reactor are in their stoichiometric proportions, that is, from reaction (1.3) they are equal.

\section{Results}

In Section 3.1 we determine the steady-state multiplicity and the double-Hopf point multiplicity. Over the parameter-region of interest there are twenty-three generic steady-state diagrams. These steady-state diagrams are discussed in Section 3.2. Where appropriate we combine steady-state analysis with numerical integration of the governing equations. We investigate the evolution of the system from the initial condition $\mathscr{I}=\left(\mathscr{F}^{*}, \mathscr{B}^{*}, \mathscr{O}_{2}^{*}, T^{*}\right)=(0,0,0,1)$.

A strong indication that a periodic orbit is terminated at a homoclinic bifurcation is if the period of the solutions increases dramatically at an accumulation point. However, in Sal'nikov systems the period of the solutions can increase dramatically at an apparent accumulation point before gradually decreasing $[2,3]$. Thus strong conclusions should not be drawn from the presence of apparent accumulation points, particularly as no Bogdanov-Takens bifurcations were found in the investigated parameter region. 


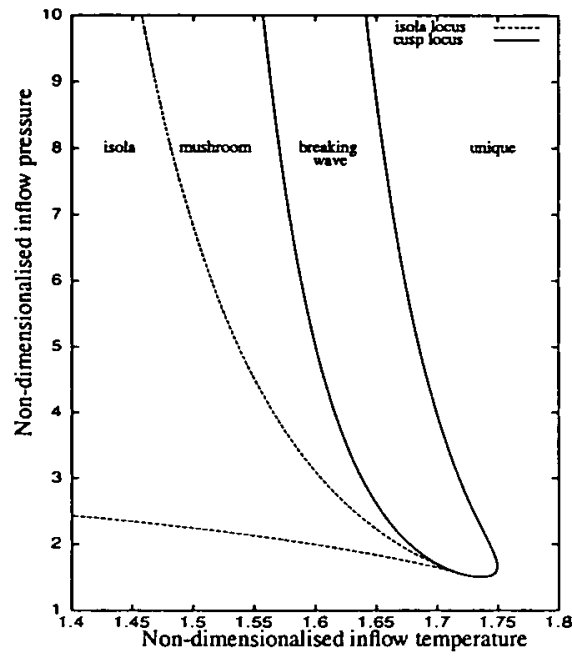

(a) Steady-state bifurcation diagram.

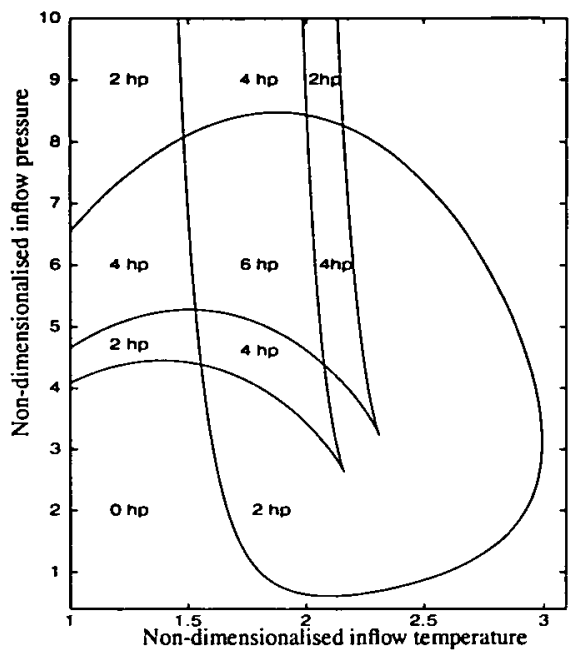

(b) Double-Hopf point bifurcation diagram.

FIGURE 1. Co-dimension 1 bifurcation diagrams.

3.1. Co-dimension 1 bifurcation diagrams Figure 1 (a) shows the steady-state bifurcation diagram. As the double-limit point singularity does not occur in our model this is determined by plotting the locus of the isola and cusp singularities. These curves divide the inflow pressure-inflow temperature (ambient temperature) plane into four regions. Thus there are four generic steady-state diagrams: a unique solution, a breaking wave solution, a mushroom and an isola. The isola and cusp locus intersect at a pitchfork singularity, a co-dimension two point. All four generic steady-state diagrams exist in a neighbourhood of this point. The pitchfork singularity is therefore an organising centre for our model.

Figure 1 (b) shows the double-Hopf point bifurcation diagram. In crossing the double-Hopf point locus two Hopf points appear or disappear in the steady-state diagram. The double-Hopf point locus divides the inflow pressure-inflow temperature plane into ten regions. The number of Hopf points within each region, marked on the figure as $n \mathrm{hp}$, is not known a priori and is found by determining a steady-state diagram in each region.

When Figure 1 (b) is overlayed onto Figure 1 (a) the resulting bifurcation diagram contains twenty-three regions. The location of these regions are shown in Figures 2-3 and are given labels. If the label is of the form $\mathrm{Aa}$ (i) then:

A defines the basic steady-state structure: I for isola, $\mathbf{M}$ for mushroom, $\mathbf{B W}$ for breaking wave and $\mathbf{U}$ for unique.

a defines the number of Hopf points in the region and takes the value $0,2,4$ or 6 .

(i) is optional and takes the value a, b or c. If there are two forms of the Aa 


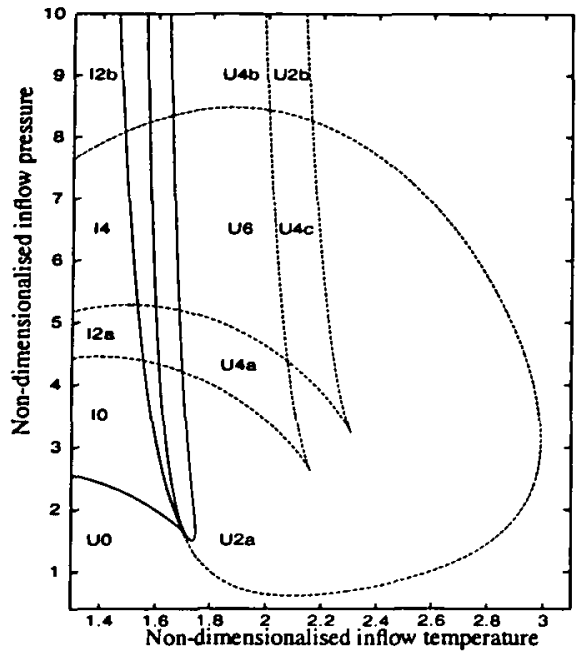

(a)

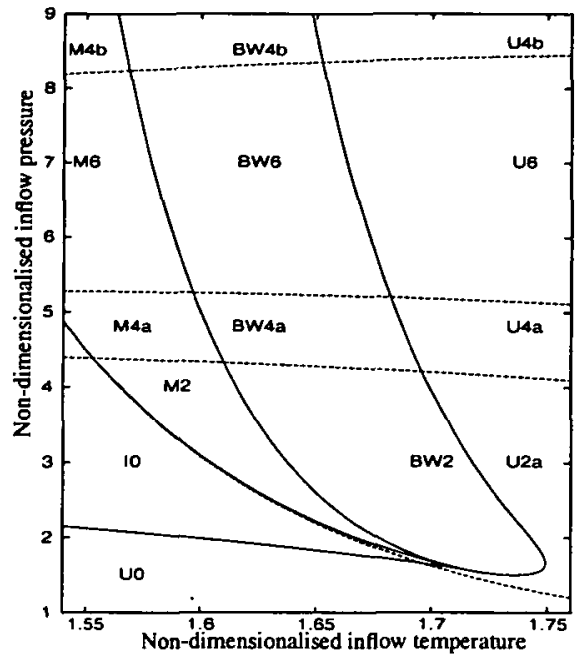

(b)

FIGURE 2. Combined bifurcation diagram obtained from Figure 1. See Figure 3 for a classification of the regions along the isola-mushroom boundary. The nomenclature is defined in Section 3.1.

steady-state diagram they are denoted $\mathrm{Aa}(\mathrm{a})$ and $\mathrm{Aa}(\mathrm{b})$. If there is a third form it is denoted $\mathrm{Aa}(\mathrm{c})$.

Hence a region labelled Ih(i)U2 refers to an isola steady-state curve where there are i Hopf points on the isola branch $(i=0,2,4,6)$ and 2 Hopf points on the unique branch. Comparing Figure 2 with Figure 3 it is evident that, with the exception of the IOU2 steady-state type, regions of the form IaUj(i) occupy a very small part of the parameter space alongside the boundary between the transition from an isola to a mushroom.

\subsection{Steady-state diagrams}

3.2.1. The isola steady-state structure Figures 4-5 show the generic isola steadystate diagrams. In four of these, shown in Figure 4, the Hopf points occur on the isola branch. In the others, shown in Figure 5, there are two Hopf points on the unique branch.

The sequences of steady-state diagrams shown in Figure 4 can arise from a unique steady-state containing no Hopf points, for example, Figure 6 (a), by increasing the inflow pressure at a fixed inflow temperature. Figure 4 (a) shows that when the boundary between the unique and isola steady states is crossed the isola is unstable. This is different from the same transition in the adiabatic reactor where the isola has stable and unstable branches, the 'upper' and 'lower' parts of the isola between the two extinction limit points respectively. In the adiabatic case the transition across the 


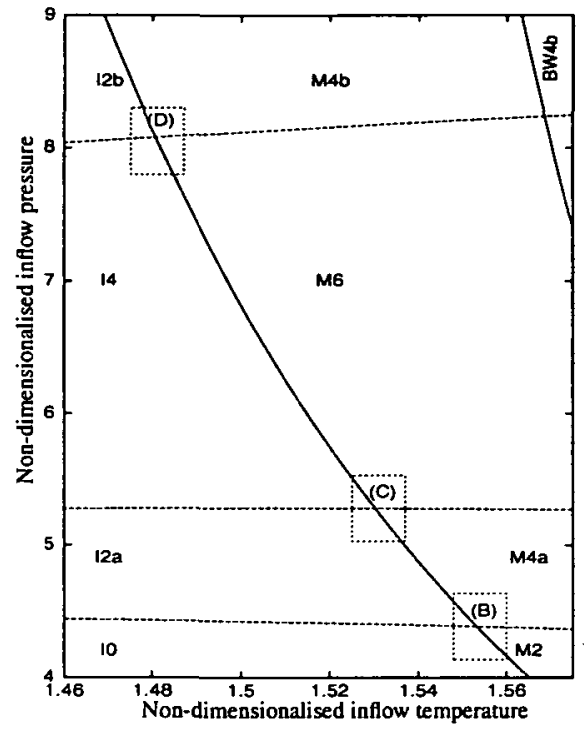

(a)

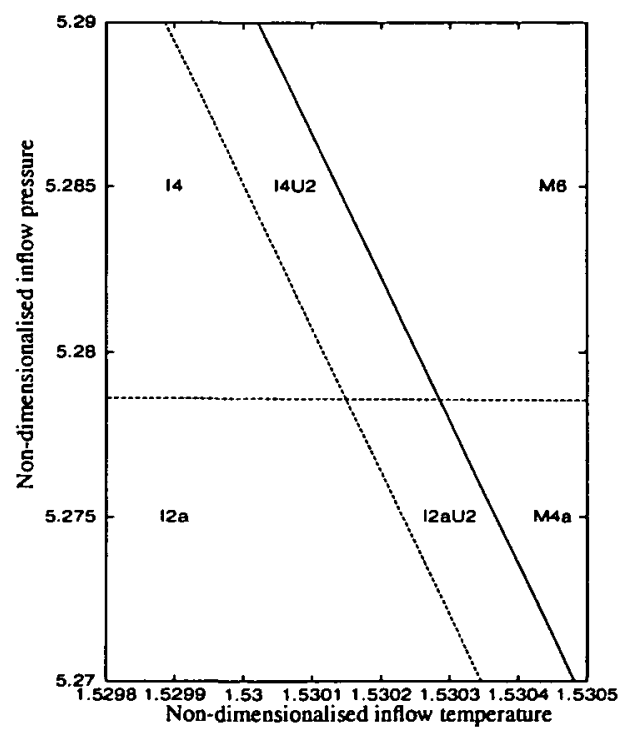

(c)

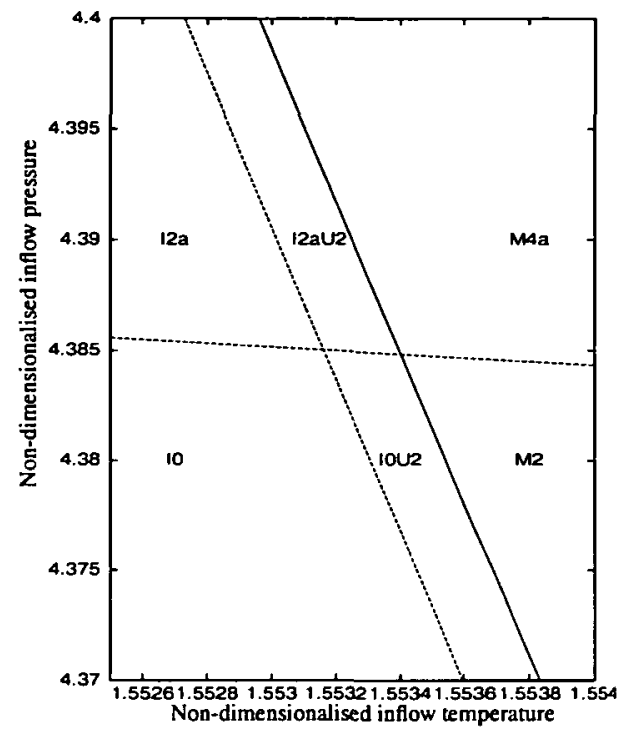

(b)

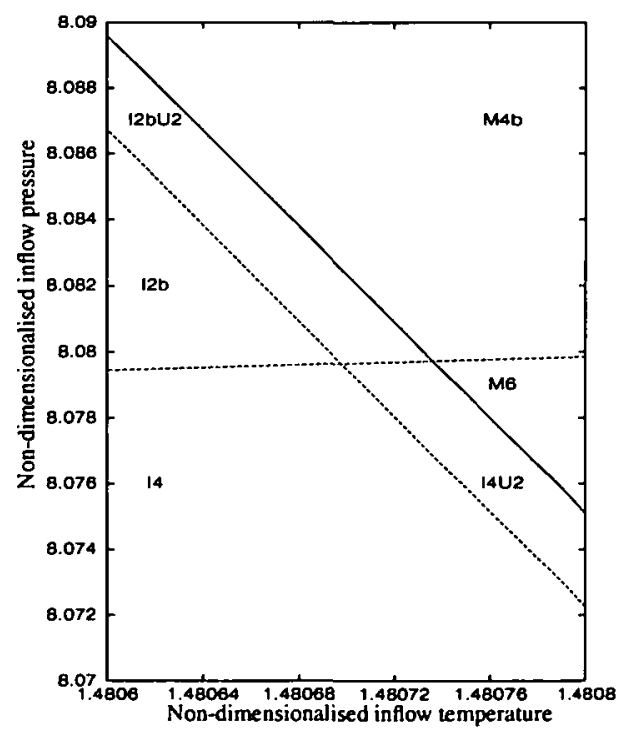

(d)

FIGURE 3. Combined bifurcation diagram along the isola-mushroom boundary. See Figure 2 for the 'large-scale' picture. The nomenclature is defined in Section 3.1. 


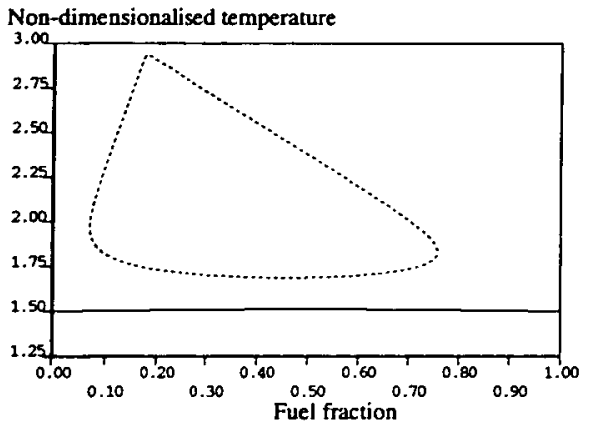

(a) 10 steady-state structure.

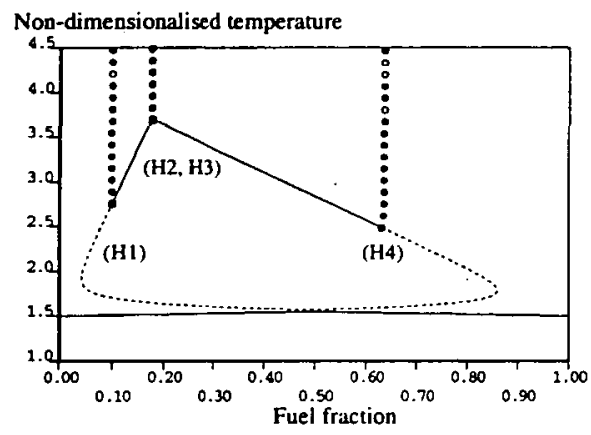

(c) I4 steady-state structure.

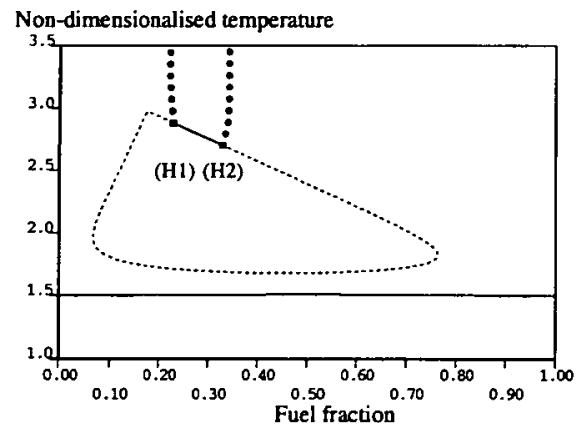

(b) I2a steady-state structure.

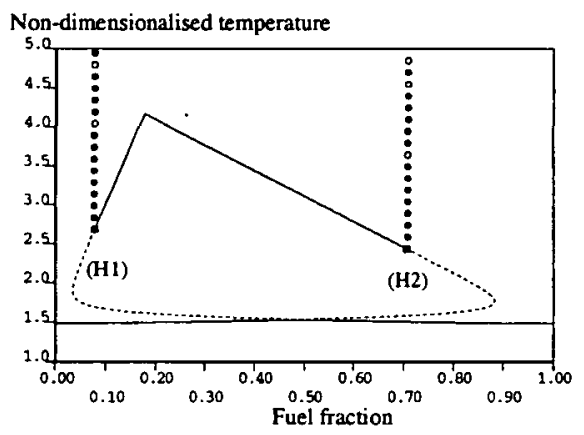

(d) I2b steady-state structure.

Figure 4. The Ia(i) steady-state structures. Parameter values are as follows. Dimensionless inflow pressure: (a) $\mathscr{P}^{*}=4.4$, (b) $\mathscr{P}^{*}=4.5$, (c) $\mathscr{P}^{*}=6.7$, (d) $\mathscr{P}^{*}=8.08$. Dimensionless inflow temperature: (a) $\mathscr{T}_{0}^{*}=1.5$, (b) $\mathscr{T}_{0}^{*}=1.5$, (c) $\mathscr{T}_{0}^{*}=1.5$, (d) $\mathscr{T}_{0}^{*}=1.48$.

isola boundary represents a change from a situation in which the air/fuel mixture is always non-flammable to one in which it is potentially flammable. This is not the case for the diabatic reactor; Figure 4 (a) represents conditions under which any fuel-air mixture is non-flammable.

As the inflow pressure is increased the system moves through a double-Hopf point. This generates the I2a steady-state structure, shown in Figure 4 (b). The double-Hopf point occurs at a fuel-rich value of the fuel fraction; the two Hopf points that are formed are therefore fuel-rich. Between the two Hopf points the 'high temperature' branch of the isola is stable. This corresponds experimentally to a region in which a stable steady flame exists. The steady-state temperature value in this region is the non-adiabatic 'flame' temperature. The region of stability is terminated at each end by a Hopf point. Both of the Hopf points are supercritical, thus as the fuel fraction is slowly decreased (increased) through the respective Hopf point stable limit cycles are formed. The maximum temperature on the limit cycle $\left(T_{m}^{*}\right)$ increases rapidly as the fuel fraction is decreased (increased) from the respective Hopf point. If we define 
a mixture to be flammable when the system corresponds to a stable flame rather than an 'oscillating flame' the flammability limits for this system are defined by the Hopf points.

As the inflow pressure is raised further a second double-Hopf point occurs, producing the $\mathrm{I} 4$ structure exhibited in Figure 4 (c). (Note that the $\mathrm{H} 2$ and $\mathrm{H} 3$ Hopf points are too close to be separated visually.) This double-Hopf point occurs at a fuel-lean value of the fuel fraction. There are now four Hopf points on the isola: three of these are fuel-rich ( $\alpha=0.1755,0.17734$ and 0.6324$)$ and one is fuel-lean $(\alpha=0.1)$. All four are supercritical and stable limit cycles emerge from them as the stable flame state loses stability. The maximum temperature on the limit cycle increases rapidly as the fuel fraction is varied away from the Hopf point. The region on the isola between the $\left(\mathrm{H}_{2}\right)$ and $(\mathrm{H} 3)$ points is unstable. Numerical integration in this region from the initial condition $\mathscr{I}$ shows that the attractor is a limit-cycle with high maximum temperature. If we define a mixture to be flammable when the system corresponds to a 'stable flame' then the I4 steady-state structure has two regions of flammability: one that predominantly represents fuel-lean mixtures $(0.1<\alpha<0.1755)$ and one that is fuel-rich $(0.17734<\alpha<0.6324)$.

At even higher values of the inflow pressure there is a third double-Hopf point. Passing through this creates the I2b steady-state structure illustrated in Figure 4 (d). At this double-Hopf point the (H3) and (H4) Hopf points in the I4 structure annihilate each other, leaving a steady-state structure containing two supercritical Hopf points. Between these two Hopf points the 'high temperature' branch of the isola is stable. Thus, as is the case in Figure 4 (b), the flammability limits for a stable flame are defined by the two Hopf points. The difference between Figure 4 (b) (I2a) and Figure 4 (d) (I2b) is that in the former the two Hopf points are both fuel-rich whereas in the latter one is fuel-lean and one is fuel-rich. As before the maximal temperature on the limit cycles increases rapidly as the fuel fraction is varied through a small parameter region.

Figure 5 shows the Ia(i)U2 steady-state diagrams. As shown in Figures 2-3 these occur in a narrow region of parameter space alongside the isola-mushroom boundary. If we follow this boundary from a low inflow pressure/'high' inflow temperature point to a high inflow pressure/'low' inflow temperature point then the transition of steady-states occurs in the order (a)-(d) presented in Figure 5. The development of the Hopf points on the isola, and their stability, follows that described above for the Ia(i) structures, shown in Figure 4. All the Hopf points on the isola in Figure 5 are supercritical. The limit cycles emerging from them initially have a small maximum temperature, but this value rapidly increases as the fuel fraction is changed very slightly.

In each of the steady-state structures shown in Figure 5 the Hopf points on the unique branch, labelled ( $\mathrm{H} 1)$ and $(\mathrm{H} 2)$, are subcritical. Unstable limit cycles emerge from them. It was only possible to continue these limit cycles over a small range in 


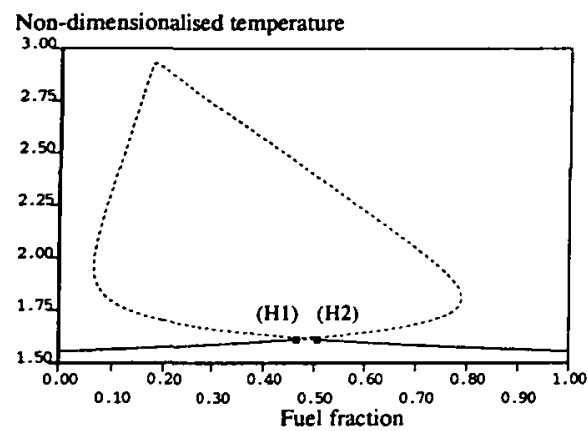

(a) IOU2 steady-state structure.

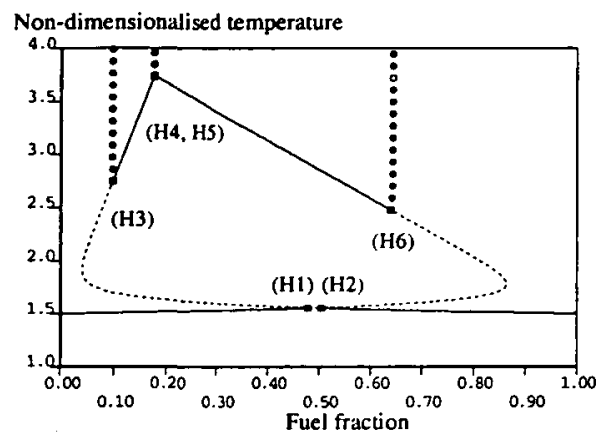

(c) I4U2 steady-state structure.

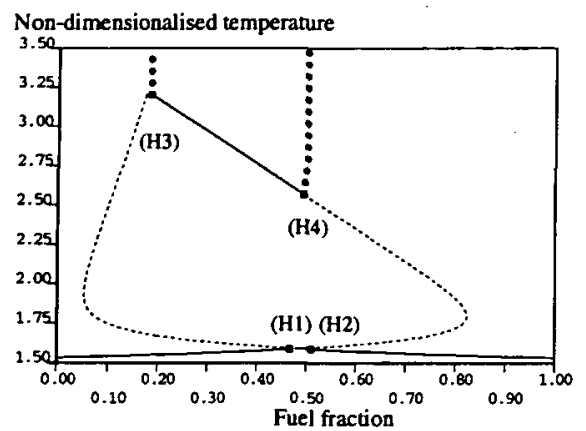

(b) I2aU2 steady-state structure.

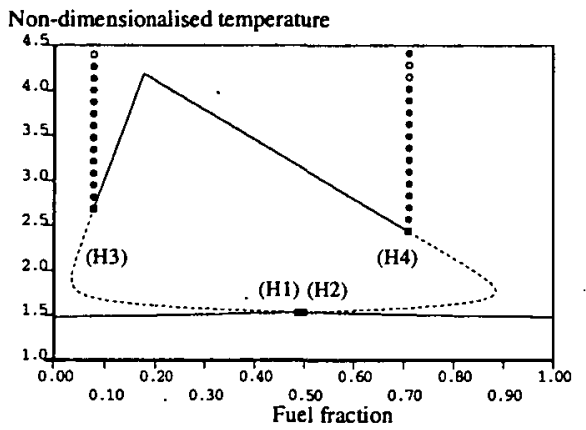

(d) I2bU2 steady-state structure.

FIGURE 5. The Ia(i)U2 steady-state structures. These diagrams contain two disjoint solution branches: an isola (containing either 0,2 or 4 Hopf points) and an unique solution branch (containing two Hopf points). Parameter values are as follows. Dimensionless inflow pressure: (a) $\mathscr{g} *=4.36$, (b) $\mathscr{P O P}^{*}=$ 5.247, (c) $\mathscr{P}^{*}=6.815$ and (d) $\mathscr{P P}^{*}=8.131$. Dimensionless inflow temperature: (a) $T_{0}^{*}=1.554$, (b) $T_{0}^{*}=1.531$, (c) $T_{0}^{*}=1.5$ and (d) $T_{0}^{*}=1.48$.

the value of the fuel fraction. Over this range the maximum temperature on the limit cycle does not increase significantly. Due to the scale of the diagrams these limit cycles are not visible in Figure 5. The period of the limit cycles increases dramatically at a possible accumulation point, typically to values of the order $10^{9}$.

In Figure 5 (a) the unique branch is unstable over the range $0.466297<\alpha<$ 0.5074747 . For values of $\alpha$ in this region integration from the initial condition $\mathscr{I}$ reveals that the system evolves onto a stable periodic solution with high amplitude ( $c a$ 400) and a period of approximately 4-5 times greater than the residence time.

In Figure 5 (b) the unique branch is unstable in the range $0.467152<\alpha<$ 0.5115688 whereas the isola is stable in the range $0.18560 \leq \alpha \leq 0.49540$. Numerical integration from the point $\mathscr{I}$ shows that when $0.47 \leq \alpha \leq 0.495$ the system evolves onto the stable flame branch on the isola. In the range $0.4954 \leq \alpha \leq 0.501$ the attractor is a limit cycle with a small amplitude. The period of the limit cycle is a 


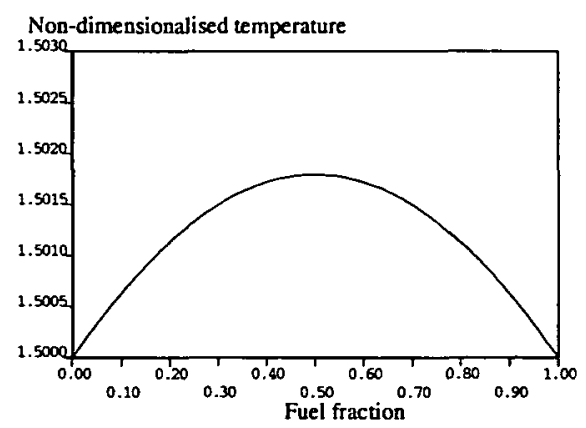

(a) UO steady-state structure.

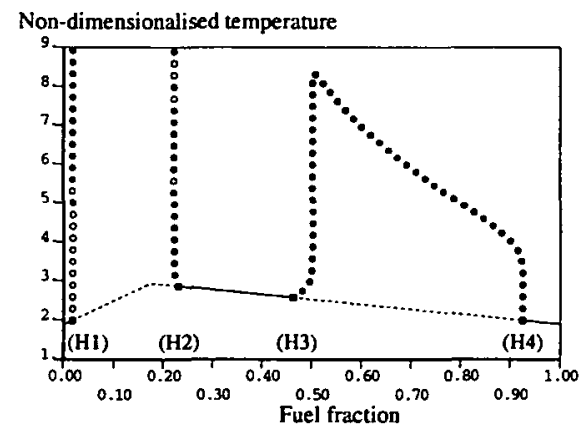

(c) U4a steady-state structure.

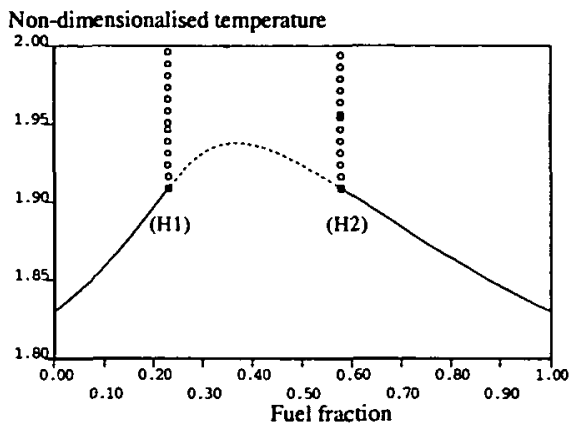

(b) U2a steady-state structure.

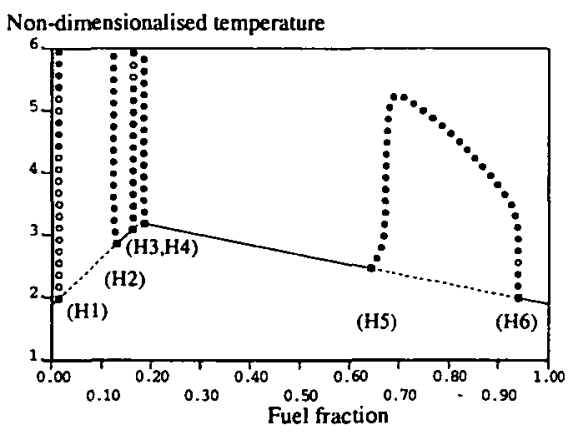

(d) U6 steady-state structure.

FIGURE 6. The Ua(i) steady-state structures. Parameter values are as follows. Dimensionless inflow pressure: (a) $\mathscr{P}^{*}=2.2$, (b) $\mathscr{P}^{*}=1.0$, (c) $\mathscr{P}^{*}=4.0$ and (d) $\mathscr{P}^{*}=5.0$. Dimensionless inflow temperature: (a) $T_{0}^{*}=1.5$, (b) $T_{0}^{*}=1.9$, (c) $T_{0}^{*}=1.9$ and (d) $T_{0}^{*}=1.9$.

small fraction of the residence time. Finally, when $0.502 \leq \alpha \leq 0.511$ the attractor is a high amplitude limit cycle. The period of the limit cycle is 5-5.5 times greater than the residence time. In both Figures 5 (c) and (d) the system evolves onto the steady flame branch from the point $\mathscr{I}$ for values of the fuel fraction for which the unique branch is unstable.

None of the steady-state structures represented in Figure 5 represent flammable mixtures in the usual sense because there are parameter values at which a stable flame does not co-exist with a stable quiescent state.

3.2.2. The unique steady-state structure Figures 6-7 show the seven generic unique steady-state diagrams. The transition U0-U2a-U4a-U6-U4b can occur at a fixed inflow temperature as the inflow pressure is increased. The transition U0-U2b$\mathrm{U} 4 \mathrm{c}$ can appear at fixed inflow temperature as the inflow pressure is increased.

Figure 6 (a) shows a unique steady-state branch containing no Hopf points. For the parameter values chosen the increase in temperature as the fuel fraction is varied 
is negligible-approximately $0.5 \mathrm{~K}$. As the inflow pressure is increased the system moves through a double-Hopf point. This occurs for a fuel-rich value of the fuel fraction, producing a steady-state diagram with two fuel-rich Hopf points. As the inflow pressure is increased the value of the fuel fraction at which the (H1) point occurs decreases through the stoichiometric fraction. This yields the steady-state diagram shown in Figure 6 (b) with a Hopf point either side of the stoichiometric fraction ( $\alpha=0.1304$ and $\alpha=0.6547$ ). Both Hopf points in this figure are subcritical. Numerical integration from the initial condition $\mathscr{I}$ shows that when the fuel fraction is in the region $0.1304<\alpha<0.6547$ the stable attractor is a limit cycle. As both of the Hopf points are subcritical, hard generation of limit cycles occurs when the fuel fraction is increased through $(\mathrm{H1})$, the maximum temperature on the limit cycle is $T_{m}^{*}=54.7$ when $\alpha=0.1304$, or decreased through (H2), $T_{m}^{*}=12.4$ when $\alpha=0.6547$. The maximum value of $T_{m}^{*}(72.6)$ is found for a slightly fuel-rich mixture $(\alpha=0.176)$.

As the inflow pressure is increased further a second double-Hopf point occurs, producing the U4a structure exhibited in Figure $6(\mathrm{c})$. The double-Hopf point occurs at a fuel-rich value of the fuel fraction. There are now four Hopf points: three supercritical fuel-rich points and one subcritical fuel-lean point. The (H2) point that was subcritical in Figure $6(\mathrm{~b})$ is now supercritical. Thus somewhere in the parameter region between Figures 6 (b) and 6 (c) the first Lyapunov coefficient of the (H2) point vanishes. As the (H1) point is subcritical, hard generation of limit cycles occurs as the fuel fraction is increased through the value $\alpha=0.0176$. As the (H2) point is supercritical, soft termination of limit cycles occurs as the fuel fraction is increased through the value $\alpha=0.2313$. The value of $T_{m}^{*}$ changes dramatically near the (H2) point. As the fuel fraction is decreased by 0.001 , from 0.222 to 0.221 , the maximum temperature increases from $T_{m}^{*}=3.44$ to $T_{m}^{*}=34.2$. Between $(\mathrm{Hl})$ and $(\mathrm{H} 2)$ the maximum value of $T_{m}^{*}$ occurs for a slightly fuel-rich mixture: $T_{m}^{*}=76.5$ when $\alpha=0.175$.

At even higher values of the inflow pressure there is a third double-Hopf point. Passing through this creates the U6 structure illustrated in Figure 6 (d). The doubleHopf point occurs at a fuel-lean value of the fuel-fraction, between the (H1) and (H2) Hopf points on Figure 6 (c). There are three fuel-lean Hopf points and three fuel-rich Hopf points on this figure. The $(\mathrm{Hl})$ point is subcritical, so that there is hard generation of limit cycles as the fuel fraction is increased through the value $\alpha=0.0136$. The other Hopf points are supercritical; soft generation/termination of limit cycles occurs at these points. The maximum temperature on the limit cycles occurs for a marginally fuel-rich mixture: $T_{m}^{*}=79.3$, when $\alpha=0.176$.

As the inflow pressure is increased the the location of the $(\mathrm{H} 4)$ bifurcation in Figure 6 (d) decreases through the stoichiometric fraction, to give a steady-state diagram with four fuel-lean Hopf points and two fuel-rich Hopf points. Following 


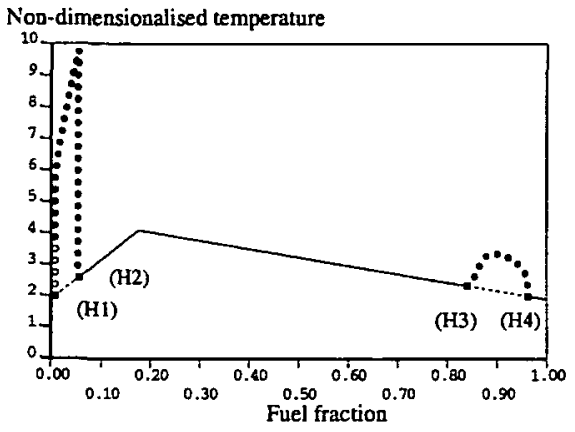

(a) U4b steady-state structure.

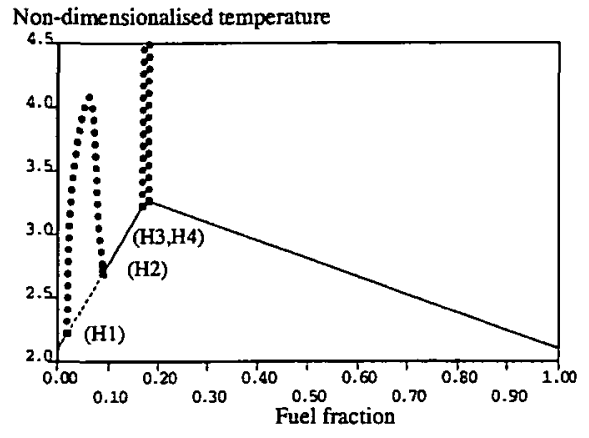

(b) U4c steady-state structure.

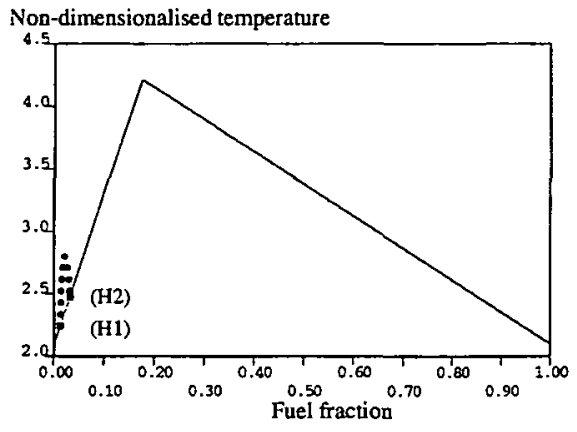

(c) U2b steady-state structure.

FIGURE 7. The Ua(i) steady-state structures. Parameter values are as follows. Dimensionless inflow pressure: (a) $\mathscr{P O}^{*}=8.5$, (b) $\mathscr{g}^{*}=5.0$ and (c) $\mathscr{g}^{*}=9.0$. Dimensionless inflow temperature: (a) $T_{0}^{*}=1.9$, (b) $T_{0}^{*}=2.1$ and (c) $T_{0}^{*}=2.1$.

this the $(\mathrm{H} 3)$ and $(\mathrm{H} 4)$ points are destroyed at a double-Hopf point. This produces Figure 7 (a), in which there are two Hopf points on either side of the stoichiometric fraction. The (H1) point is subcritical whilst the others are supercritical. Thus there is hard generation of limit cycles as the fuel fraction is increased through (H1) and soft generation/termination at $(\mathrm{H} 2-\mathrm{H} 4)$. Again there is a dramatic change in the maximal temperature in the vicinity of the $(\mathrm{H} 2)$ point. The maximum temperature on the limit cycles occurs for a fuel-lean mixture: $T_{m}^{*}=9.825$ when $\alpha=0.053$.

The U4c structure shown in Figure 7 (b) can be formed in various ways. Starting with a U6 structure, for example, Figure 6 (d), we can fix the inflow pressure and increase the inflow temperature. The $(\mathrm{H} 5)$ and $(\mathrm{H} 6)$ points in Figure 6 (d) are destroyed at a double-Hopf point producing the U4c steady-state structure. This has four supercritical Hopf points, three fuel-lean and one fuel-rich. Note that the $(\mathrm{H} 1)$ point that was subcritical in Figure 6 (d) is now supercritical. Thus somewhere in the parameter region between Figure 6 (d) and Figure 7 (b) the first Lyapunov coefficient of this Hopf point vanishes. In the vicinity of each Hopf point the maximum temperature 


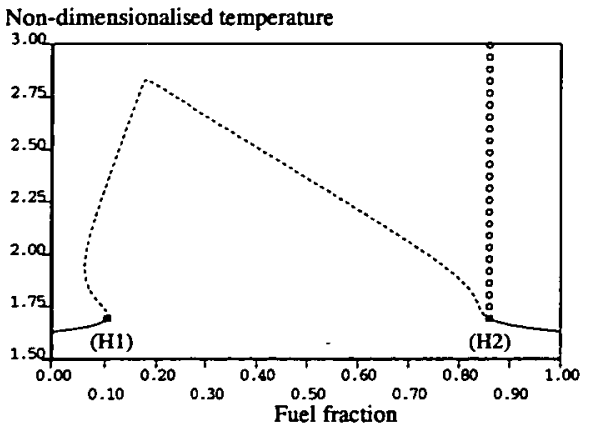

(a) BW2 steady-state structure.

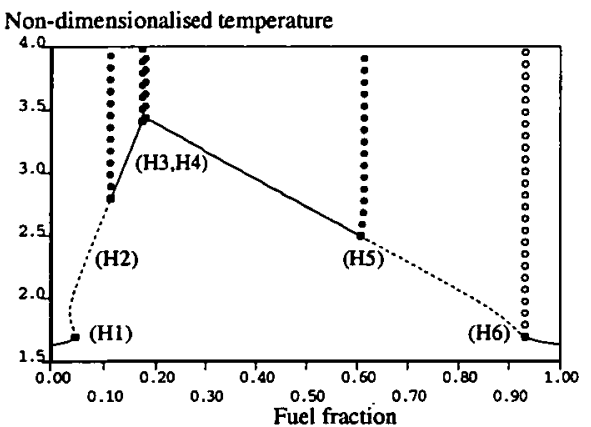

(c) BW6 steady-state structure.

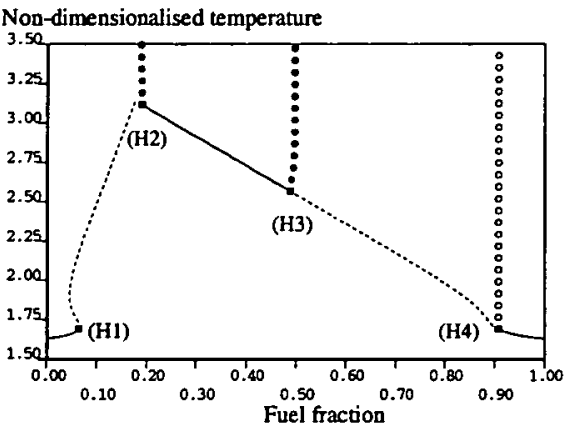

(b) BW4a steady-state structure.

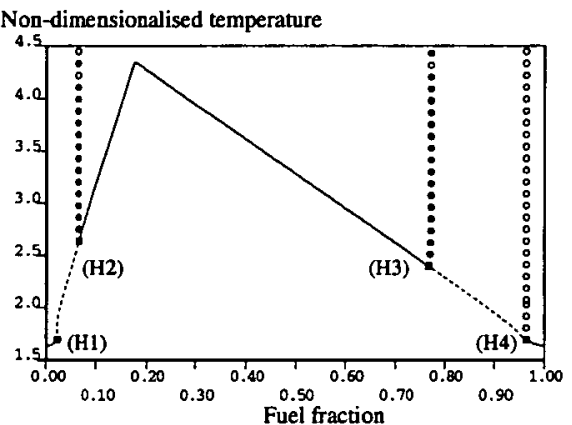

(d) BW4b steady-state structure.

FIGURE 8. The BWa(i) steady-state structures. Parameter values are as follows. Dimensionless inflow pressure: (a) $\mathscr{P}^{*}=4.0$, (b) $\mathscr{P}^{*}=5.0$, (c) $\mathscr{P}^{*}=6.0$ and (d) $\mathscr{P}^{*}=9.0$. Dimensionless inflow temperature: (a) $T_{0}^{*}=1.63$, (b) $T_{0}^{*}=1.63$, (c) $T_{0}^{*}=1.63$ and (d) $T_{0}^{*}=1.63$.

on the limit cycle changes dramatically over a small range of the fuel fraction. The maximum value of $T_{m}^{*}$ is found for a slightly fuel-rich mixture, $T_{m}^{*}=31.7$ when the fuel fraction is $\alpha=0.17644$.

The U2b steady-state structure, Figure 7 (c), is produced from the U4c steadystate structure, Figure 7 (b), by a double-Hopf point that destroys the (H3) and (H4) bifurcation points. Both the remaining Hopf bifurcations are supercritical. Observe that the maximum value of $T_{m}^{*}$ is now lower than the maximum stable flame temperature.

3.2.3. The breaking wave structure Figure 8 shows the four generic breaking wave steady-state diagrams. Figure 2 shows that the transition BW2-BW4a-BW6-BW4b can occur at a fixed inflow temperature as the inflow pressure is increased. In each of these steady-state structures no limit cycles are shown emerging from the (H1) Hopf point. Over the parameter range in which it was possible to continue the limit cycles, the maximum temperature $\left(T_{m}^{*}\right)$ did not increase significantly. Due to the scale of the 
diagram these limit cycles are not visible in Figure 8. The period of the limit cycles increases dramatically at an accumulation point, typically to values of the order of $10^{8}$.

In Figure 8 (a) the breaking wave solution loses stability at the two Hopf points $(\alpha=0.1058$ and 0.8610 ). Numerical integration shows that between these values the system evolves onto a limit cycle with high maximum temperature. In both cases hard generation of limit cycles occurs as the steady state loses stability. Moving through the fuel-lean Hopf point the maximum temperature on the limit cycle is approximately 330 . This increases to a maximum value of 459 at a marginally fuelrich value of the fuel fraction $(\alpha=0.177)$, decreasing to a value of 70 as the system increases through the fuel-rich Hopf point.

As the inflow pressure is increased the system moves through a double-Hopf point. This occurs at a fuel-rich value of the fuel fraction, creating two supercritical fuelrich Hopf points. This transition creates the BW4a steady-state structure shown in Figure 8 (b). The four Hopf points on this figure occur at $\alpha=0.0658,0.1907,0.489$ and 0.908 . As the fuel fraction is increased through the $(\mathrm{H1})$ point, hard generation of limit cycles occurs: $T_{m}^{*}=252$ when $\alpha=0.066$. As the fuel fraction is increased $T_{m}^{*}$ increases to a maximum value of 460 at a fuel-rich composition ( $\alpha=0.181$ ). The maximum temperature then decreases very rapidly over a small parameter range: when $\alpha=0.189, T_{m}^{*}=434$ but when $\alpha=0.19, T_{m}^{*}=3.3$. As the fuel fraction is increased through (H2) there is soft termination of stable limit cycles. Increasing the fuel fraction through the supercritical $(\mathrm{H} 3)$ point sees the soft generation of limit cycles, although the value of $T_{m}^{*}$ increases rapidly as the fuel fraction is subsequently increased. For instance, when $\alpha=0.490$ the maximum temperature is $T_{m}^{*}=2.613$, whereas when the fuel fraction is $\alpha=0.50$ the maximum temperature is $T_{m}^{*}=125$. As the fuel fraction is increased from 0.5 towards the $(\mathrm{H} 4)$ point the maximum temperature decreases. As the fuel fraction is increased through (H4) there is hard termination of the limit cycles with $T_{m}^{*}=55$ at $\alpha=0.907$.

As the inflow pressure is raised further a second double-Hopf point occurs, producing the BW6 structure exhibited in Figure 8 (c). The double-Hopf point generates two fuel-lean Hopf points. In Figure 8 (c) there are three Hopf points on the fuel-lean side $(\alpha=0.04559,0.1135$ and 0.1733$)$ and three on the fuel-rich side $(\alpha=0.1793,0.6075$ and 0.9323 ). As the fuel fraction is increased through ( $\mathrm{H} 1)$ hard generation of limit cycles occurs: $T_{m}^{*}=203$ when the fuel fraction is $\alpha=0.04559$. As the fuel fraction is increased $T_{m}^{*}$ increases to a maximum value, $T_{m}^{*}=304$, at a fuel-lean composition $(\alpha=0.111)$. As shown in Figure 8 (c) the maximum temperature then decreases dramatically over a small parameter region as the periodic solution branch terminates at a supercritical Hopf bifurcation (H2): $T_{m}^{*}$ decreases from 304 to 3.0 , as the fuel fraction increases from $\alpha=0.111$ to $\alpha=0.112$. As the fuel fraction is increased through (H3) soft generation of limit cycles occurs, although the maximum temperature increases dramatically: from $T_{m}^{*}=3.514$ to $T_{m}^{*}=460.2$, as the fuel fraction is increased from 


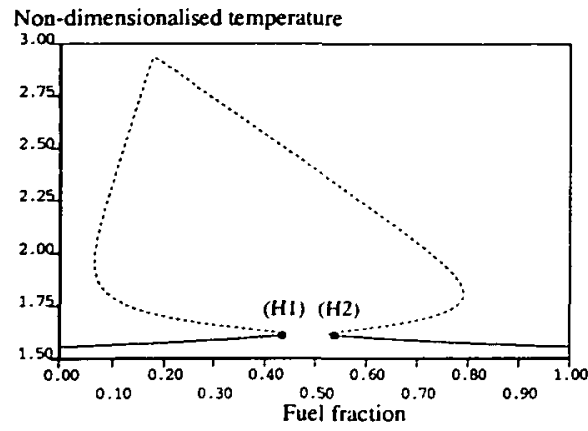

(a) M2 steady-state structure.

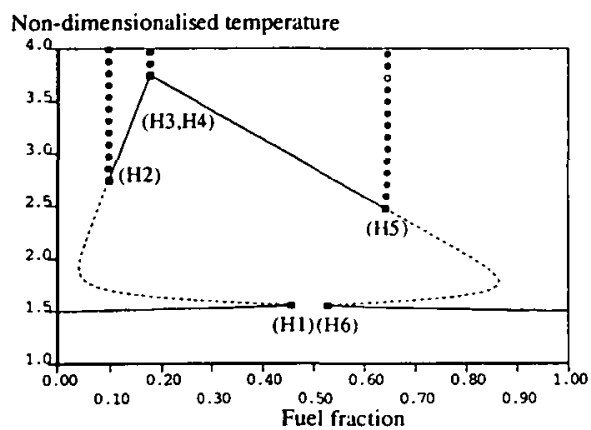

(c) M6 steady-state structure.

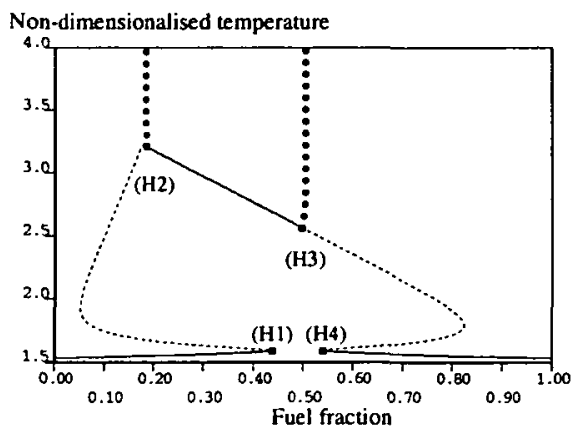

(b) M4a steady-state structure.

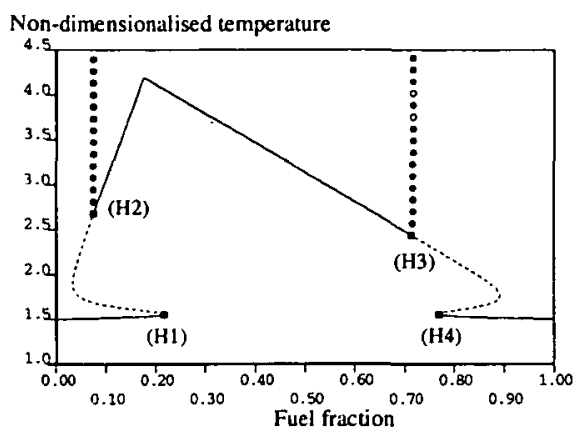

(d) M4b steady-state structure.

FIGURE 9. The Ma(i) steady-state structures. Parameter values are as follows. Dimensionless inflow pressure: (a) $\mathscr{P}^{*}=4.38$, (b) $\mathscr{P}^{*}=5.27$, (c) $\mathscr{P}^{*}=6.83$ and (d) $\mathscr{P O}^{*}=8.2$. Dimensionless inflow temperature: (a) $T_{0}^{*}=1.554$, (b) $T_{0}^{*}=1.531$, (c) $T_{0}^{*}=1.5$ and (d) $T_{0}^{*}=1.5$.

$\alpha=0.1733$ to $\alpha=0.174$. Between (H3) and (H4), $T_{m}^{*}$ takes its maximum value (466.4) at a marginally fuel-rich value of the fuel fraction $(\alpha=0.176)$. The maximum temperature then decreases over a very small range of the fuel fraction $\left(T_{m}^{*}=460\right.$ when $\alpha=0.179, T_{m}^{*}=4.02$ when $\alpha=0.1791$ ) as the limit cycles are destroyed at a supercritical Hopf bifurcation (H4). As the fuel fraction is increased through (H5) soft generation of limit cycles occurs. Again there is a small region where the maximum temperature changes rapidly, increasing from $T_{m}^{*}=2.8$ when $\alpha=0.614$ to $T_{m}^{*}=82.7$ when $\alpha=0.615$. The maximum temperature then decreases as the fuel fraction is increased through (H6), where there is hard termination of the limit cycles $\left(T_{m}^{*}=44.6\right.$ when $\alpha=0.932$ ). The maximum temperature for the fuel-rich limit cycles between (H5) and (H6) is significantly lower than the marginally fuel-lean/fuel-rich values between ( $\mathrm{H} 3)$ and $(\mathrm{H} 4)$.

At even higher values of the inflow pressure there is another double-Hopf point. Prior to this the marginally fuel-lean Hopf point, (H3) in Figure 8 (c), increases through the stoichiometric value of the fuel fraction. The double-Hopf point destroys 
the two middle Hopf points, creating the BW4b structure shown in Figure 8 (d). This is the first breaking wave structure in which the steady-state solution with the highest temperature is stable. This figure contains two fuel-lean Hopf points $(\alpha=0.0209$ and 0.0065 ) and two fuel-rich Hopf points $(\alpha=0.7684$ and 0.9643$)$. As the fuel fraction is increased through (H1) there is hard generation of limit cycles $\left(T_{m}^{*}=131\right.$ when $\alpha=0.0210)$. The amplitude increases to a maximum $\left(T_{m}^{*}=179.7\right.$ when $\left.\alpha=0.064\right)$ and then decreases dramatically $\left(T_{m}^{*}=2.715\right.$ when $\left.\alpha=0.065\right)$ as the limit cycles are terminated supercritically at $(\mathrm{H} 2)$. As the fuel fraction is increased through $(\mathrm{H} 3)$ there is soft generation of stable limit cycles, but the maximum temperature rapidly increases: $T_{m}^{*}=2.48$ when $\alpha=0.770$, but $T_{m}^{*}=43.6$ when $\alpha=0.780$. The maximum temperature then decreases as the fuel fraction is increased through $(\mathrm{H} 4)$, where there is hard termination of the limit cycles $\left(T_{m}^{*}=29.2\right.$ when $\left.\alpha=0.964\right)$. The maximum temperature on the limit cycles appears to be lower on the fuel-rich side than the fuel-lean side.

3.2.4. The mushroom structure Figure 9 shows the four generic mushroom steadystate diagrams. The transition M2-M4a can arise at a fixed inflow temperature as the inflow pressure is increased (see Figure 2). The transition M4a-M6-M4b can arise at a fixed inflow temperature as the inflow pressure is increased. In Figures 9 (a)(d) no limit cycles are shown emerging from two Hopf points. In each case the maximum temperature of the limit cycle did not increase significantly over the small parameter range in which it was possible to continue them. Due to the scale of the diagram these limit cycles are not visible in Figure 9. The period of these limit cycles increases dramatically at an accumulation point. For some values of the fuel fraction the mushroom structure does not contain a 'low-valued' (no-ignition) stable steady state. For values of the fuel fraction in this region we determined the evolution of the system from the initial condition $\mathscr{I}$ by integration.

Figure 9 (a) illustrates the M2 steady-state structure. There are subcritical Hopf points at $\alpha=0.4359$ and $\alpha=0.5380$. Between these points no part of the steadystate structure is stable. As the fuel fraction is increased through $\alpha=0.4359(\mathrm{H} 1)$ there is hard generation of limit cycles, with a maximum temperature $T_{m}^{*}=445.4$. The maximum temperature decreases as the fuel fraction is increased. There is hard termination of the limit cycles at $\alpha=0.538(\mathrm{H} 2)$, where the maximum temperature is $T_{m}^{*}=359.9$.

As the inflow pressure is increased the system moves through a double-Hopf point. This generates the M4a steady-state structure, shown in Figure 9 (b). This bifurcation creates two 'high-valued' fuel-rich supercritical Hopf points. These occur at $\alpha=0.1852$ and $\alpha=0.4986$. Between (H3) and (H4) the 'steady-flame branch' of the mushroom is stable. The two 'low-valued' Hopf points are at $\alpha=0.4388$ and $\alpha=0.5405$. When the fuel fraction is in the range $0.4388<\alpha \leq 0.4986$ the system evolves onto the stable high-temperature branch. The (H3) Hopf point is 
supercritical and when the fuel fraction is increased through 0.4986 the attractor is a small-amplitude limit-cycle. The maximum temperature rapidly increases: $T_{m}^{*}=2.82$ when $\alpha=0.505$, but $T_{m}^{*}=476.1$ when $\alpha=0.506$. As the fuel fraction is increased further the maximum temperature of the limit cycles decreases slightly. As the fuel fraction is increased through the (H4) point the system is attracted to the 'low-valued' branch.

As the inflow pressure is raised further a second double-Hopf point occurs, producing the M6 steady-state structure exhibited in Figure 9 (c). This has six Hopf points. Two of these are on the 'low-temperature' branch, at $\alpha=0.4571$ and $\alpha=0.5279$, and four on the 'high-temperature' branch, at $\alpha=0.0978, \alpha=0.175674, \alpha=0.1772$ and $\alpha=0.6414$. The latter are all supercritical. When the fuel fraction is in the range $0.4571<\alpha<0.5279$ the system evolves onto the 'high-valued' stable steady-state branch.

At even higher values of the inflow pressure there is a third double-Hopf point. At this point the (H3) and (H4) Hopf points in the M6 structure are destroyed. This creates the steady-state structure shown in Figure 9 (d). This contains two 'low-valued' Hopf points, at $\alpha=0.2184$ and $\alpha=0.7691$ and two 'high-valued' supercritical Hopf points, at $\alpha=0.0771$ and $\alpha=0.7149$. Note that the steady state at which the maximum stable temperature occurs is now stable. For values of the fuel fraction in the region $0.2184<\alpha \leq 0.7149$ the system is attracted to the stable 'steady-flame' branch. As the fuel fraction is increased through $\alpha=0.7149$, corresponding to (H3), the stable flame loses stability to low-amplitude limit cycles. The amplitude of these limit cycles rapidly increases over a small parameter region. As the fuel fraction is increased from 0.718 to 0.719 the maximum temperature increases from $T_{m}^{*}=2.686$ to $T_{m}^{*}=382.8$. As the fuel fraction is increased further the maximum temperature slowly decreases, to $T_{m}^{*}=350$ when the fuel fraction is 0.7691 . When the fuel fraction is 0.7692 , or higher, the system evolves onto the 'low-temperature' steady-state branch.

\section{Discussion}

The low temperature oxidation of hydrocarbons generates a rich variety of phenomena including steady-state multiplicity, birhythmicity, cool flames, oscillatory ignition, two-stage and multi-stage ignition, complex ignition and steady ignition [5]. An interesting question is to what extent this range of behaviour can be generated by simple chemical models. The simple mechanism used in this paper generates steady-state multiplicity and steady-ignition. Our focus is the issue of flammability as the fuel fraction is varied. The I2a, I4 and I2b steady-state structures, illustrated in Figure 4, offer an explanation of flammability in a CSTR. The stable flame branch is disjoint from the no-ignition branch so that a perturbation on the system is required 


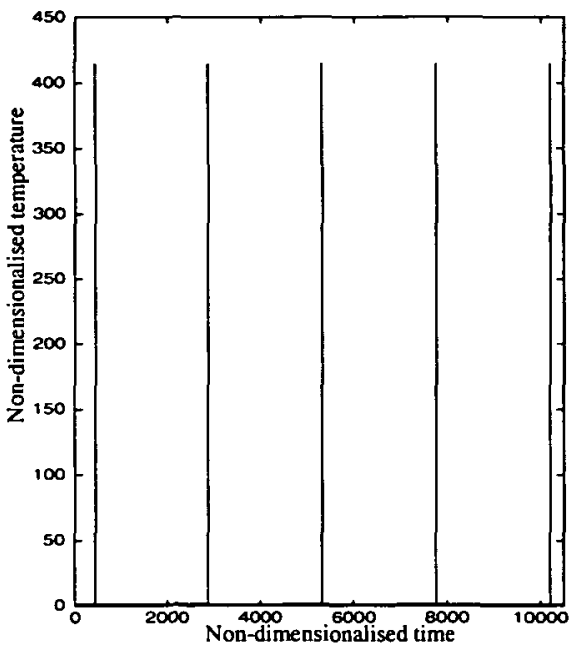

(a)

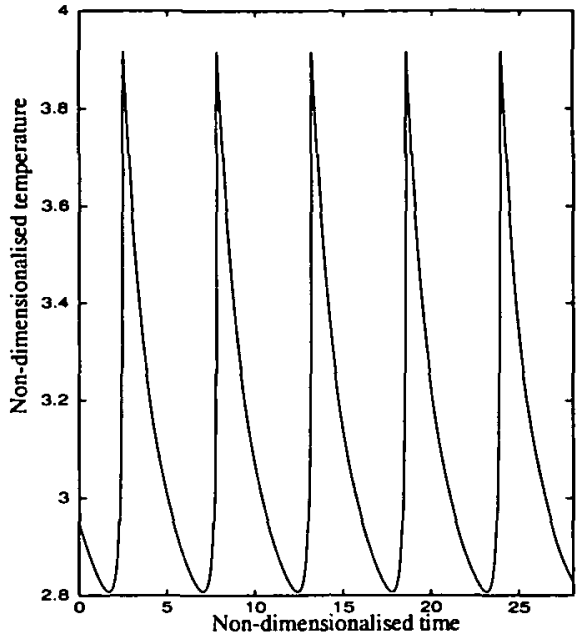

(b)

FIGURE 10. Oscillatory ignition (a) and cool flames (b). Parameter values (a): fuel fraction, $\alpha=0.47$; dimensionless inflow pressure, $\mathscr{P}^{*}=4.36$; dimensionless inflow temperature, $T_{0}^{*}=1.554$. Parameter values (b): Fuel fraction, $\alpha=0.1641$; dimensionless inflow pressure, $\mathscr{P}^{*}=5$; dimensionless inflow temperature, $T_{0}^{*}=1.9$.

to ignite a flammable mixture. Furthermore a stable flame can only be formed if the fuel fraction lies within sharply defined limits.

Although the 12aU2, I4U2 and I2bU2 steady states, shown in Figure 5, contain a stable flame branch that is disjoint from the no-ignition branch these structures do not represent flammable mixtures because the no-ignition branch is not stable for all values of the fuel fraction. Consequently for some values of the fuel fraction a steady flame can be formed without a perturbation as the steady-flame state is not bistable with a quiescent state. In some circumstances a steady flame can be formed by smoothly changing the fuel fraction without a discontinuous response. For instance in the $U 4 c$ and $U 2 b$ steady-state structures, depicted in Figure 7, a steady-flame state is formed smoothly as the the fuel fraction is decreased. (There is a smooth response in the steady-state temperature to changes in the fuel fraction.) In such circumstances a critical value of the fuel fraction can be identified using the concept of sensitivity analysis [7]. There are also steady-fiame states in the breaking wave, Figure 8, and mushroom, Figure 9, steady-state diagrams.

The dynamics of our model are governed by a two-dimensional submodel. As a result neither two-stage/multi-stage ignition nor chaotic behaviour can occur. However, both oscillatory ignition and cool flames are exhibited. The key features of oscillatory ignition are that the period of the limit cycle is greater than the residence time, although usually it is of the same order of magnitude, and that the maximum temperature is 
many hundreds of kelvin. The flow-rates used in our simulations correspond to a residence time of approximately 495 dimensionless time units. The time series for the limit cycle shown in Figure 10 (a) represents simple oscillatory ignition with a period approximately 5 times the residence time. This figure illustrates that simple oscillatory ignition is a relaxation oscillation. Cool flame oscillations are a characteristic of the oxidation reactions of many hydrocarbon fuels. A particularly important effect of this mode of reaction is that of engine knock, which occurs during the heating of petrol-air mixtures in the compression stage of the internal combustion cycle. The temperature excursions may be as small as a few kelvin, but usually are of the order of $100-150 \mathrm{~K}$ (which is only a fraction of the maximum or adiabatic flame temperature). Figure 10 (b) shows a cool-flame oscillation with a period approximately 0.011 of the residence time.

In the adiabatic reactor flammability limits are defined by the extinction limit points on the isola [7]. For the parameter values considered in this paper the flammability limits in the I2a, I4 and I2b structures are defined by Hopf bifurcation points. When there are no Hopf points the isola is entirely unstable and the degenerate double-Hopf bifurcation not only creates Hopf points it also effectively forms regions where the stable-flame state exists. In Figure 4 (d) there is a fuel-rich limit and a fuel-lean limit. In contrast Figure 4 (b) shows the two flammability limits are both fuel-rich whereas in Figure 4 (c) there are two sets of flammability limits. These diagrams say no more than that flammability limits are not an intrinsic property of a fuel, they depend upon the conditions under which they are measured.

Although the main emphasis of this paper is on flammability, the steady-state diagrams also have implications for the operation of chemical reactors. For instance, it is important to ensure that large amplitude temperature oscillations do not occur as they are detrimental to reactor integrity (thermal cycling). It is instructive to compare Figure 7 (b) with Figure 7 (c). In the former, oscillations occur between the Hopf points $(\mathrm{H} 3)$ and $(\mathrm{H} 4)$ with large variations in the temperature. This region of parameter space should therefore be avoided. However, if the reactor is being run on the stable flame branch near to the $(\mathrm{H} 4)$ point a small decrease in the fuel fraction, possibly caused by a down-stream perturbation, could result in large amplitude oscillations. On the other hand high-valued temperature limit cycles are not found in Figure 7 (c) and small perturbations on the fuel fraction can not destabilise the reactor in this way. Interestingly, the inflow pressure is higher in Figure 7 (c) than in Figure 7 (b). This is insightful because whilst it seems "obvious" that it would be more "dangerous" to operate at higher rather than lower pressure, in some circumstances the reverse is the case.

As the fuel fraction is varied the maximum steady-state flame temperature always occurs at a slightly fuel-rich mixture. If this solution is unstable the fuel fraction at which the maximum stable-flame temperature is found corresponds to a fuel-rich 
Hopf point. In most of the steady-state diagrams the maximum value of $T_{m}^{*}$ is higher than the maximum stable-flame temperature. This is certainly true for the cases depicted in Figures 4 (b)-(d), 5 (a)-(d), 6 (b)-(d), 7 (a)-(b), 8 (a)-(d) and 9 (a)-(d). In some circumstance the highest value of $T_{m}^{*}$ is found for a reactant composition that is fuel-lean, for example, Figure 7 (a).

\section{Conclusion}

In this paper we have extended a previous study into the dynamics of a single oxidative Sal'nikov scheme in an adiabatic continuously stirred tank reactor [7] to a diabatic reactor. In both the adiabatic and diabatic reactor the static steady-state multiplicity has a pitchfork singularity as an organising centre.

The inclusion of heat loss increases the dimensionality of the system from one to two, opening the possibility of periodic behaviour due to Hopf bifurcations. We have investigated the creation of Hopf bifurcations via the double-Hopf point bifurcation. When the steady-state multiplicity and the degenerate Hopf point bifurcation diagrams are combined they give rise to a total of twenty-three generic steady-state diagrams. For our purposes it suffices to know the number of Hopf points on a given steadystate diagram. The number of generic regions would be higher if we were to consider whether each Hopf point was either subcritical or supercritical (the Bautin bifurcation). An alternative approach would be to classify by the number of Hopf points on each steady-state structure that are fuel-lean/fuel-rich. Our classification of these structures illustrates the power of the 'global singularity theory method'. This method not only determines the number of generic steady-state diagrams but defines their region of existence. Some of these regions are very small and could be missed experimentally.

Having established the generic steady-state diagrams in a diabatic reactor we are presently exploring how this framework can be used to investigate the action of gasphase active fire retardants in a continuously-stirred tank reactor.

\section{Acknowledgements}

During this work MIN was supported by a grant from the the Australian Research Council.

\section{References}

[1] E. J. Doedel, T. F. Fairgrieve, B. Sandstede, A. R. Champneys, Y. A. Kuznetsov and X. Wang, AUTO 97: Continuation and bifurcation software for Ordinary Differential Equations (with HomCont), March 1998. Available by anonymous fip from ftp.cs. concordia.ca/pub/doedel/auto. 
[2] L. K. Forbes, "Limit-cycle behaviour in a model chemical reaction: the Sal'nikov thermokinetic oscillator", Proc. Roy. Soc. London Ser. A 430 (1990) 641-651.

[3] L. K. Forbes, M. R. Myerscough and B. F. Gray, "On the presence of limit-cycles in a model exothermic chemical reaction: Sal'nikov's oscillator with two temperature-dependent reaction rates", Proc. Roy. Soc. London Ser. A 435 (1991) 591-604.

[4] B. F. Gray and M. J. Roberts, "A method for the complete qualitative analysis of two coupled ordinary differential equations dependent on three parameters", Proc. Roy. Soc. London Ser. A 416 (1988) 361-389.

[5] P. Gray and S. K. Scott, "Experimental systems 2: Gas-phase reactions", in Chemical oscillations and instabilities: non-linear chemical kinetics, Volume 21 of International Series of Monographs on Chemistry, 1st ed., (Clarendon Press, Oxford, 1990) Ch. 15.

[6] Y. A. Kuznetsov, Elements of applied bifurcation theory, Applied Mathematical Sciences 112, $1 \mathrm{st}$ ed. (Springer, New York, 1995).

[7] M. I. Nelson and H. S. Sidhu, "Bifurcation phenomena for an oxidation reaction in a continuously stirred tank reactor. I Adiabatic operation.", J. Math. Chem. 31 (2) (2002) 155-186.

[8] I. Ye. Sal'nikov, "A thermokinetic model of homogeneous periodic reactions", Dokl. Akad. Nauk SSSR 60 (3) (1948) 405-408, in Russian.

[9] I. Ye. Sal'nikov, "Contribution to the theory of the periodic homogeneous chemical reactions: II A thermokinetic self-excited oscillating model", Zh. Fiz. Khimii 23 (1949) 258-272, in Russian.

[10] M. J. Sexton and L. K. Forbes, "An exothermic chemical reaction with linear feedback control", Dynam. Stability Systems 11 (3) (1996) 219-238. 